\title{
Dielectric studies of dispersions of carbon nanotubes in liquid crystals 5 CB
}

\author{
A. Koval'chuk, L. Dolgov, O. Yaroshchuk \\ Institute for Physics, NAS of Ukraine, 46, prospect Nauky, 03028 Kyiv, Ukraine
}

\begin{abstract}
The frequency dependences of the imaginary $\varepsilon^{\prime \prime}$ and real $\varepsilon^{\prime}$ parts of complex dielectric permittivity inherent to planarly aligned layers of nematic liquid crystals $5 \mathrm{CB}$ doped with multiwalled carbon nanotubes (CNT) were investigated in a wide range of frequencies $\left(f=10^{-2}-10^{6} \mathrm{~Hz}\right)$ and CNT concentrations $(c=0-0.25 \mathrm{wt} . \%)$. It has been shown that the studied frequency range can be divided in three parts according to behavior of $\varepsilon^{\prime}(f)$ and $\varepsilon^{\prime \prime}(f)$ curves. The low-frequency range $\left(10^{-2}<f<10^{1} \mathrm{~Hz}\right)$ reflects the near-electrode processes in the cell. The ratio $\varepsilon^{\prime \prime} / \varepsilon^{\prime}$ used to characterize these processes sharply grows if the concentration of CNT exceeds $0.05 \mathrm{wt} . \%$. The moderate frequency range $\left(10^{1}<f<10^{5} \mathrm{~Hz}\right)$ corresponds to the alternating current conductivity, $\sigma_{\mathrm{AC}}$. At the nanotubes concentration less than $0.025 \mathrm{wt} . \%, \sigma_{\mathrm{AC}}$ does not depend on the frequency that implies its ionic origin. In its turn, at the $c \geq 0.025 \mathrm{wt} . \%, \sigma_{\mathrm{AC}}$ is a power function of the frequency that is typical for electronic hopping mechanism. The transition from the ionic to electronic conductivity can be explained by the percolation theory with the critical concentration of nanotubes $0.031 \mathrm{wt} . \%$ and percolation parameter 2.5 . The high-frequency range $\left(10^{5}<f<10^{6}\right)$ is mainly attributed to dipole volume polarization. For $c<0.05$ wt.\% such polarization is well described by the Debye equation. The time of dielectric relaxation in this frequency range increases with nanotubes content. This is explained by effective interaction of nanotubes with $5 \mathrm{CB}$ molecules.
\end{abstract}

Keywords: liquid crystal, carbon nanotube, permittivity, conductivity.

Manuscript received 18.06.08; accepted for publication 20.10.08; published online 11.11.08.

\section{Introduction}

Influence of nanoparticles on the properties of liquid crystals (LC) is topical and attracts stable scientific interest [1]. Among the variety of nanoparticles used to fill LC, carbon nanotubes (CNT) take a special place. Because of elongated shape, the nanotubes can be easily built into the orientationally ordered liquid crystal matrix $[2,3]$. This leads to a number of interesting effects, such as reduction of controlling voltage and time of electrooptic response [4-6], elimination of image sticking [7] and appearance of electro-optic memory [8]. All these effects are related with dielectric properties of LC-CNT composites.

In spite of big importance of dielectric characteristics for understanding of remarkable properties of LC-CNT dispersions, only few studies were focused on this problem. Dierking et al. [2,3] and Huang et al. [6] revealed, correspondingly, enhancement of anisotropy of electric conductivity and dielectric constant of LC-CNT dispersions due to orientational ordering of CNT in nematic LC. Lee et al. [4] described influence of CNT on the structure of double electric layers formed near electrodes in the LC cells. David et al. [9] presented results of theoretical calculations of dielectric losses of thin LC layers adsorbed on CNT. Finally, Lebovka et al. [10] revealed sharp increase of electric conductivity with CNT concentration, which is caused by formation of continuous network of CNT in LC matrix.

This work is focused on frequency dependences of real, $\varepsilon^{\prime}$, and imaginary, $\varepsilon^{\prime \prime}$, parts of dielectric permittivity (dielectric spectra) of LC-CNT dispersions. According to our earlier studies [11-13], this approach allows to distinguish different processes of dielectric relaxation, improve understanding structural organization and interfacial interaction in the LC-CNT systems. Besides, it is important from the methodological point of view, because it gives a correct frequency range to measure electrical conductivity. By analyzing dielectric spectra, 
we clarify changes in charge transfer mechanisms and electric field distribution within LC cells realized by insertion of CNT.

\section{Materials and methods}

As a host material we used nematic liquid crystal npentyl-n'-cyanobiphenyl (5CB) from Merck, Germany, with dielectric anisotropy $\Delta \varepsilon=11.7$ and range of nematic mesophase $22.5-35.5^{\circ} \mathrm{C}$. It is a well studied liquid crystal frequently used as a reference $\mathrm{LC}$ media.

To fill 5CB, we used the multiwalled carbon nanotubes from SpecMash Ltd., Ukraine. They were prepared from ethylene by the chemical vapor deposition method [14]. Typically, these CNT have outer diameter about $12-20 \mathrm{~nm}$ and length about tens of micrometers. The specific surface area $S$ of the tubes is $190 \mathrm{~m}^{2} / \mathrm{g}$ $( \pm 10 \%)$. The specific electric conductivity $\sigma$ of the powder of compressed multiwalled CNT (at pressure $15 \mathrm{TPa}$ ) is $10.0 \mathrm{~S} / \mathrm{cm}$ along the axis of compression and appears to be significantly lower than that of the graphite $\left(\sigma=10^{3}-10^{6} \mathrm{~S} / \mathrm{cm}[15]\right)$.

Liquid crystal $5 \mathrm{CB}$ was mixed with nanotubes by ultrasonic mixer УЗДН-2Т (Russia). The mixing time was $20 \mathrm{~min}$. We investigated series of samples with a varied content of nanotubes: $0 ; 0.025 ; 0.05 ; 0.1$ and 0.25 wt. $\%$.

The glass substrates with transparent conductive indium tin oxide coatings and aligning polyimide layers were used for the assembling of liquid crystal cells. We used polyimide SE150 (JSR, Japan) designed for planar LC alignment. The polyimide layers were unidirectionally rubbed by a fleecy cloth. The cells were assembled so that rubbing directions of different substrates were antiparallel. The thickness of the cells was set by $16 \mu \mathrm{m}$ glass spacers. These cells were filled capillary with pristine LC or LC doped with nanotubes.

From the electric viewpoint, LC cells can be presented by an equivalent circuit consisting of the parallel connected resistance $R$ and capacity $C$. The dependences of $R$ and $C$ on the frequency $f$ of the sinelike testing electric field applied across the LC cell were measured by the oscilloscopic method [16]. The amplitude of the measured signal was $0.25 \mathrm{~V}$. The frequency $f$ was varied within the range $5 \cdot 10^{-2}-10^{6} \mathrm{~Hz}$. Using the measured data of $R$ and $C$, real $\varepsilon^{\prime}$ and imaginary $\varepsilon^{\prime \prime}$ parts of permittivity were calculated. The temperature of samples was stabilized during measurements at $294 \pm 0.2{ }^{\circ} \mathrm{C}$.

\section{Experimental results and discussion}

The frequency dependences of $\varepsilon^{\prime}$ and $\varepsilon^{\prime \prime}$ for the planarly aligned layer of $5 \mathrm{CB}$ and $5 \mathrm{CB}-\mathrm{CNT}$ dispersion $(c=$ 0.25 wt. \%) are presented in Figs 1a and 1b, respectively. One can see that doping of a small amount of CNT essentially changes dielectric spectra of 5CB. The studied frequency range can be divided in subintervals
A $\left(10^{-2}-10 \mathrm{~Hz}\right), \quad B \quad\left(10-10^{5} \mathrm{~Hz}\right)$ and $\mathrm{C}\left(10^{5}-10^{6} \mathrm{~Hz}\right)$ according to three types of behavior of $\varepsilon^{\prime}(f)$ and $\varepsilon^{\prime \prime}(f)$ curves reflecting different processes in the cells. Below, these processes are considered one after another.

\section{A. Influence of carbon nanotubes on the near-electrode parameters}

As we cleared up earlier $[11,17]$, dielectric spectra of liquids in the frequency range A $\left(10^{-2}-10 \mathrm{~Hz}\right)$ were determined by the near-electrode processes and particularly by the charge exchange between the electrode and ions presented in the liquid. Comparing dielectric spectra of pure 5CB and 5CB doped with CNT (0.25 wt.\%) one can observe essential increase of $\varepsilon^{\prime \prime}$ value by insertion of CNT (greater than 300 times at $0.1 \mathrm{~Hz}$ ). The increase of $\varepsilon^{\prime}$ is less pronounced (less than 1.5 times at $0.1 \mathrm{~Hz}$ ). Drastic increase of $\varepsilon^{\prime \prime}$ value may be explained by increase of electronic conductivity in the near-electrode layer. It may be caused only by participation of CNT in the charge transfer. Thus, nanotubes provide an effective electron exchange between the electrodes and ions of 5CB. In other words, CNT shunt the highly resistant double electric layers preventing effective charge transport from LC bulk to electrodes. It is very likely that this effect causes lowering the controlling voltage and response time of LC by insertion of CNT $[4,5]$.

According to the discussion above, the ratio $\varepsilon^{\prime \prime} / \varepsilon^{\prime}$ can be chosen as a parameter characterizing shunting of double electric layers by CNT. The ratio $\varepsilon^{\prime \prime} / \varepsilon^{\prime}$ at fixed frequency $f=0.1 \mathrm{~Hz}$ was estimated for dispersions with various concentrations of CNT. These data are summarized in Table. It is evident that at $c<0.05 \mathrm{wt} . \%$ the parameter $\varepsilon^{\prime \prime} / \varepsilon^{\prime}$ is less than unity and so the nearelectrode charge transfer in 5CB-CNT dispersions is caused by the liquid crystal component. The nearelectrode areas are characterized by high resistivity because of formation of double electric layers hampering transport of bulk ions to electrodes. A sharp, more than two times, increase of $\varepsilon^{\prime \prime} / \varepsilon^{\prime}$ is observed within the range $0.05<c<0.25 \mathrm{wt} . \%$. This means that shunting the double electric layers is achieved in this range of CNT concentrations.

Table. Parameters of 5CB-CNT dispersions for various concentrations of carbon nanotubes.

\begin{tabular}{|c|c|c|c|c|c|}
\hline $\begin{array}{c}\text { CNT } \\
\text { concentra- } \\
\text { tion } \\
c, \text { wt. } \%\end{array}$ & $\begin{array}{c}\varepsilon^{\prime \prime} / \varepsilon^{\prime} \\
(f= \\
0.1 \mathrm{~Hz}),\end{array}$ & $\begin{array}{c}\sigma_{i}, \\
\mathrm{Ohm}^{-1} \cdot \mathrm{m}^{-1}\end{array}$ & $p$ & $\begin{array}{c}\sigma_{\mathrm{AC}} \\
(f= \\
\left.10^{3} \mathrm{~Hz}\right), \\
\mathrm{Ohm}^{-1} \cdot \mathrm{m}^{-1}\end{array}$ & $\begin{array}{c}\tau, \\
\mu \mathrm{s}\end{array}$ \\
\hline 0 & 0.59 & $7.4 \cdot 10^{-8}$ & 0 & $7.4 \cdot 10^{-8}$ & 0.56 \\
\hline 0.025 & 0.74 & $4.3 \cdot 10^{-8}$ & 0 & $4.3 \cdot 10^{-8}$ & 0.72 \\
\hline 0.05 & 0.78 & $1.9 \cdot 10^{-7}$ & 0.05 & $2.6 \cdot 10^{-7}$ & 0.94 \\
\hline 0.1 & 9.1 & $1.2 \cdot 10^{-7}$ & 0.23 & $8.6 \cdot 10^{-7}$ & \\
\hline 0.25 & 133 & $2.8 \cdot 10^{-7}$ & 0.52 & $9.9 \cdot 10^{-6}$ & \\
\hline
\end{tabular}



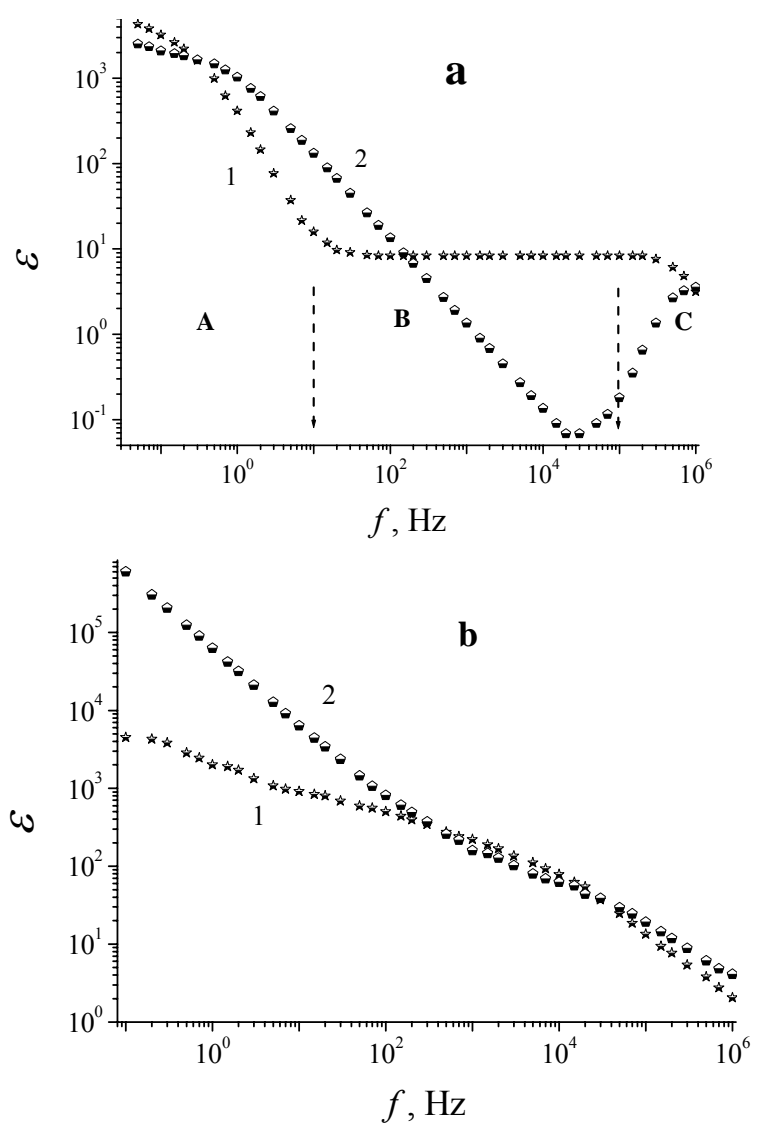

Fig. 1. Permittivity components $\varepsilon^{\prime}$ and $\varepsilon^{\prime \prime}$ as functions of the frequency of testing electric field $f$ for the pristine $5 \mathrm{CB}$ (a) and $5 \mathrm{CB}$ doped with $0.25 \mathrm{wt} . \%$ of carbon nanotubes (b). Curves 1 and 2 correspond to real $\varepsilon^{\prime}$ and imaginary $\varepsilon^{\prime \prime}$ components of the permittivity, respectively.

\section{B. Influence of carbon nanotubes on the conductivity of samples}

According to $[11,12]$, dielectric spectra in the middle frequency region $\mathrm{B}\left(10-10^{5} \mathrm{~Hz}\right)$ characterize bulk properties of the sample. The resistance $R$ and capacity $C$ in this frequency range are not dependent on the frequency for neat LC and CNT doped LC as well. It allows us to calculate the alternating current conductivity $\sigma_{\mathrm{AC}}$ :

$$
\sigma_{\mathrm{AC}}=\varepsilon_{0} \varepsilon^{\prime \prime} \omega
$$

where $\varepsilon_{0}$ is the dielectric constant, and $\omega=2 \pi f$ is the cyclic frequency.

A family of frequency dependences of $f \varepsilon^{\prime \prime}$ for the $5 \mathrm{CB}-\mathrm{CNT}$ dispersions with a different content of CNT is presented in Fig. 2. It is obvious that the value $f \varepsilon^{\prime \prime}$, and thus $\sigma_{\mathrm{AC}}$, is independent on $f$ at $c \leq 0.025 \mathrm{wt} . \%$. On the other hand, at $c>0.025 \mathrm{wt} . \%$ the alternating current conductivity $\sigma_{\mathrm{AC}}$ becomes a power function of the frequency $f$. It can be described by the formula

$$
\sigma_{\mathrm{AC}}=\sigma_{i}+s f_{n}^{m},
$$

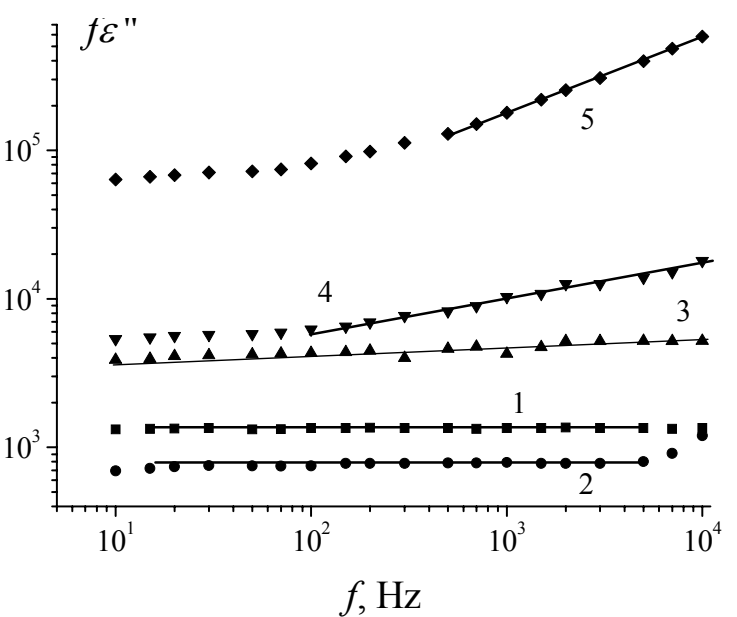

Fig. 2. Dependence of the value $f \varepsilon^{\prime \prime}$ on the frequency $f$ for the $5 \mathrm{CB}$ based dispersions with various concentrations of carbon nanotubes: 0 (1); 0.025 (2); 0.05 (3); 0.1 (4); 0.25 wt.\% (5).

where $m$ and $s$ are constants, $f_{n}$ is the normalized frequency. The first frequency independent term in (2) is typical for the ionic conductivity of liquids $\sigma_{i}$. The second term, according to [17], describes the hopping charge transfer. The latter can be attributed to electronic charge transfer through nanotubes. This assumes that the distance of hopping is a minimal distance between the neighboring nanotubes. In this case, parameter $m$ must be dependent on the concentration $c$.

The values of $\sigma_{i}$ and $m$ estimated for different concentrations of CNT are presented in Table. One can see that parameter $m$ demonstrates strong growth with CNT concentration. At $c \leq 0.025 \mathrm{wt} . \%$ parameter $m=0$ and so the ionic charge transfer mechanism, i.e., charge transfer through liquid crystal, dominates. In its turn, within the concentration range $0.05<c<0.25 \mathrm{wt} . \%$, the parameter $m$ increases more than one order of magnitude. This implies strong growth of electronic conductivity described by the term 2 in the formula (2). So, the major mechanism of conductivity changes within the range $0.05<c<0.25 \mathrm{wt} \%$ from the ionic to electronic one.

Dependence of $5 \mathrm{CB}$ conductivity on the $\mathrm{CNT}$ concentration at $f=10^{3} \mathrm{~Hz}$ is plotted in Fig. 3 and tabulated in Table. One can see that $\sigma_{\mathrm{AC}}$ rapidly grows within the range $0.05<c<0.25 \mathrm{wt} . \%$ reflecting transition from the ionic to electronic conductivity. Similar behavior of $\sigma_{\mathrm{AC}}(c)$ curve for other LC-CNT system was described in [10]. The observed concentration transition of conductivity was considered as percolation transition caused by formation of continuous network of CNT in LC media.

According to [19], in case of percolation, the $\sigma_{\mathrm{AC}}(c)$ curve should be extrapolated by

$\sigma_{\mathrm{AC}}=k\left(c-c_{\mathrm{cr}}\right)^{p}$

where $k$ is the coefficient of proportionality, $c_{\mathrm{cr}}$ is the critical concentration, and $p$ is the parameter of 


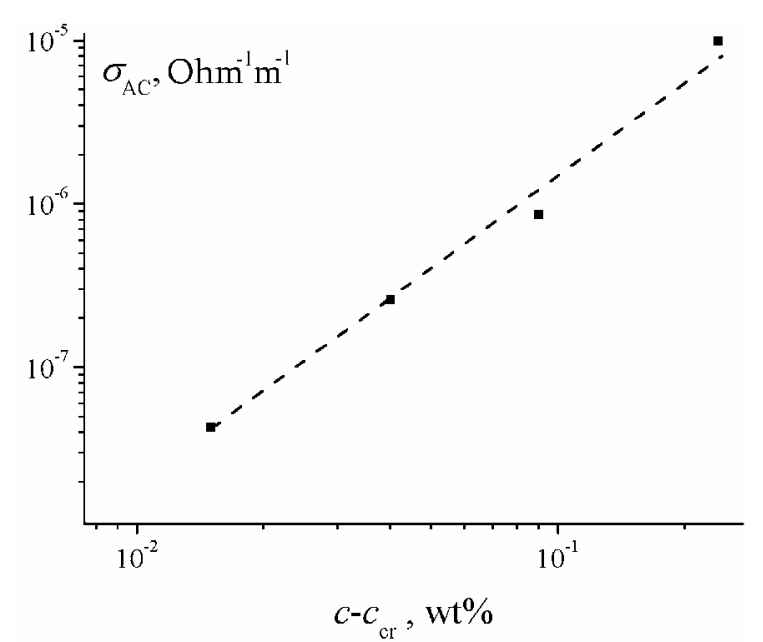

Fig. 3. Conductivity of $5 \mathrm{CB}-\mathrm{CNT}$ dispersion vs the concentration of nanotubes. $f=10^{3} \mathrm{~Hz}$.

percolation. From Fig. 3, one can see that the equation (3) fits the experimental points very well at $c_{\mathrm{cr}}=0.01 \mathrm{wt} . \%$ and $p=1.9$. This additionally confirms our assumption about percolation behavior of $\sigma_{\mathrm{AC}}$ in LCCNT dispersions.

\section{Influence of the carbon nanotubes on the bulk dipole polarization}

The frequency region $\mathrm{C}\left(10^{5}-10^{6} \mathrm{~Hz}\right)$ of dielectric spectra (Fig. 1) characterizes dipole polarization of the sample bulk. The frequency dependences of $\varepsilon^{\prime}$ and $\varepsilon^{\prime \prime}$ for such polarization are described by the Debye equation [20]:

$\varepsilon^{*}=\varepsilon_{\infty}+\frac{\varepsilon_{s}-\varepsilon_{\infty}}{1+i \omega \tau}$

where $\varepsilon^{*}$ is the complex permittivity, $\varepsilon_{s}$ and $\varepsilon_{\infty}$ correspondingly are permittivities for the frequencies $f=0$ and $f \rightarrow \infty, \tau$ is the time of dielectric relaxation.

To verify whether the measured spectra in region $\mathrm{C}$ obey the equation (4), the Cole-Cole diagrams $\varepsilon^{\prime \prime}\left(\varepsilon^{\prime}\right)$ were plotted. The corresponding diagram for $5 \mathrm{CB}$ doped with 0.05 wt.\% of CNT is presented in Fig. 4. It is evident that the experimental data are well fitted by a semicircle. This implies Debye character of dielectric relaxation. The same is true for all concentrations lower than $0.05 \mathrm{wt} . \%$. This is, however, not the case for higher concentrations, when the electronic conductivity of samples clearly prevails.

Since dielectric spectra for concentrations $c \leq 0.05$ wt.\% obey the Debye law (4), the time of dielectric relaxation for these concentrations can be estimated. The obtained data are presented in Table. It is evident that $\tau$ increases with increasing $c$. The reason of this may be strong interaction between LC and nanotubes [21] and growth of rotational viscosity with CNT concentration [6].

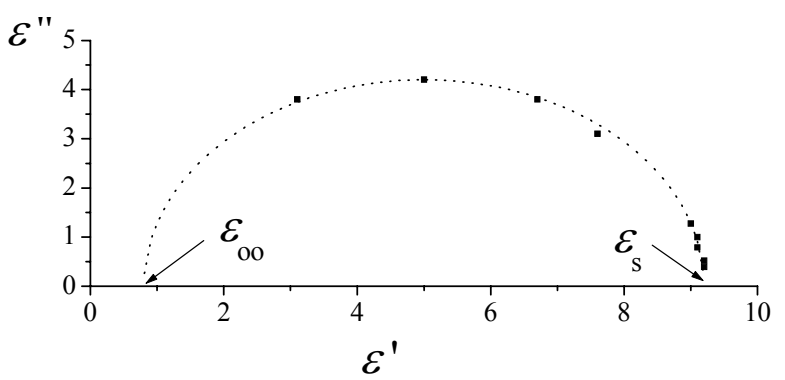

Fig. 4. Cole-Cole diagram $\varepsilon^{\prime \prime}\left(\varepsilon^{\prime}\right)$ for the $5 \mathrm{CB}$ doped with 0.05 wt. $\%$ of carbon nanotubes.

\section{Conclusions}

Frequency dependences of permittivity (dielectric spectra) for the planarly aligned layers of nematic LC $5 \mathrm{CB}$ doped with carbon nanotubes are measured and analyzed. In these spectra three frequency regions can be selected.

The region A $\left(10^{-2}-10 \mathrm{~Hz}\right)$ characterizes the nearelectrode processes. At $c>0.05 \mathrm{wt} . \%$, carbon nanotubes shunt the double electric layers drastically increasing their conductivity.

The region B $\left(10-10^{5} \mathrm{~Hz}\right)$ characterizes bulk processes, particularly, an alternating current conductivity $\sigma_{\mathrm{AC}}$. At $c \leq 0.025 \mathrm{wt} . \%, \sigma_{\mathrm{AC}}$ is independent of the frequency of electric field that implies ionic mechanism of conductivity associated with LC phase. At higher concentrations of CNT, $c>0.05 \mathrm{wt} . \%$, the $\sigma_{\mathrm{AC}}$ is a power function of the frequency that corresponds to the electronic hopping conductivity introduced by CNT. The exponent of $f$ changes more than one order of magnitude by increasing the CNT concentration from 0.05 to $0.25 \mathrm{wt} . \%$. The transition from the ionic to electronic conductivity may be described in frame of the theory of percolation. The critical concentration of percolation and percolation parameter are $0.01 \mathrm{wt} . \%$ and 1.9 , respectively.

The region $\mathrm{C}\left(10^{5}-10^{6} \mathrm{~Hz}\right)$ characterizes dipole polarization in the bulk of the dispersion layer. If $c<0.05 \mathrm{wt} . \%$, the dielectric spectra in the region $\mathrm{C}$ are well described by the Debye equation. It is shown that the time of dielectric relaxation increases with CNT concentration. This suggests strong interaction between LC molecules and CNT as well as viscosity grow. The spectra for $c \geq 0.05$ wt.\% cannot be fitted by the Debye equation due to essential electron polarization of samples introduced by carbon nanotubes.

\section{References}

1. H. Stark, Physics of colloidal dispersions in nematic liquid crystals // Phys. Repts 351, p. 387474 (2001).

2. I. Dierking, G. Scalia, P. Morales, D. LeClere, Aligning and reorienting carbon nanotubes by nematic liquid crystals // Adv. Mater. 16, No. 11, p. $865-869$ (2004). 
3. I. Dierking, G. Scalia, P. Morales, Liquid crystalcarbon nanotube dispersions // J. Appl. Phys. 97(4), 044309 (2005).

4. W. Lee, C.-Yu. Wang, and Yu-C. Shih, Effects of carbon nanosolids on the electro-optical properties of a twisted nematic liquid-crystal host // Appl. Phys. Lett. 85 (4), p. 513-515 (2004).

5. W. Lee and Yu-C. Shih, Effects of carbonnanotube doping on the performance of a $\mathrm{TN}-$ LCD // Journal of the SID, 13(9), p. 743-747 (2005).

6. C.Y Huang, C.Y. Hu, H.C. Pan and K.Y. Lo, Electrooptical responses of carbon nanotubedoped liquid crystal devices // Jpn. J. Appl. Phys. 44 (11), p. 8077-8081 (2005).

7. In-Su Baik, S.Y. Jeon, S.H. Lee, K.A. Park, S.H. Jeong, K.H. An, Y.H. Lee, Electrical field effect on carbon nanotubes in a twisted nematic liquid crystal cell // Appl. Phys. Lett. 87, 263110 (2005).

8. L. Dolgov, O. Yaroshchuk, M. Lebovka, Effect of electro-optical memory in the liquid crystals doped with carbon nanotubes // Accepted for Mol. Cryst. Liq. Cryst.

9. A. Dawid, Z. Gburski, Dielectric relaxation of 4cyano-4-n-pentylbiphenyl (5CB) thin layer adsorbed on carbon nanotube - MD simulation // J. Non-cryst. Solids 353, p. 4339-4343 (2007).

10. N. Lebovka, T. Dadakova, L. Lysetskiy, O. Melezhyk, G. Puchkovska, T. Gavrilko, J. Baran, M. Drozd, Phase transitions, intermolecular interactions and electrical conductivity behavior in carbon multiwalled nanotubes/nematic liquid crystal composites // Accepted for J. Mol. Struct.

11. A.V. Koval'chuk, Low frequency spectroscopy as investigation method of the electrode-liquid interface // Functional Materials 5(3), p. 428-430 (1998).
12. A.V. Koval'chuk, Relaxation processes and charge transport across liquid crystal-electrode interface // J. Phys.: Condens. Mater. 13(24), p. 10333-10345 (2001).

13. O. Yaroshchuk, A. Kovalchuk, R. Kravchuk, The interfacial dipole-to-dipole interaction as a factor of polar anchoring in the cells with planar liquid crystal alignment // Mol. Cryst. Liq. Cryst. 438, p. $195-204$ (2005).

14. A. Melezhyk, Yu. Sementsov, V. Yanchenko, Synthesis of porous carbon nanofibers on catalysts fabricated by the mechanochemical method // Zhurnal prikladnoy khimii 78(7), p. 924-930 (2005) (in Russian).

15. Carbon Nanotubes: Properties and Applications, ed. by M.J. O'Connell. Teylor and Francis, London, 2006.

16. A.J. Twarowski, A.C. Albrecht, Depletion layer in organic films: Low frequency measurements in polycrystalline tetracene // J. Chem. Phys. 20(5), p. 2255-2261 (1979).

17. A.V. Koval'chuk, Low-frequency dielectric relaxation at the tunnel charge transfer across the liquid/electrode interface // Functional Materials 8(4), p. 690-693 (2001).

18. N.F. Mott, E.A. Davis, Electronic Processes in Non-crystalline Materials. Clarendon Press, Oxford, 1971.

19. D Stauffer, A. Aharony, Introduction to Percolation Theory, Second ed. London, Washington DC, 1992.

20. W. Haase, S. Wrobel, Relaxation Phenomena. Springer, New York, 2003.

21. K.A. Park, S.M. Lee, S.H. Lee, and Y.H. Lee, Anchoring a liquid crystal molecule on a singlewalled carbon nanotube // J. Phys. Chem. C 111, p. $1620-1624$ (2007). 